\title{
Creencias sobre la escritura y su enseñanza en maestros peruanos de educación primaria ${ }^{1}$
}

\section{Beliefs about writing and its teaching in Peruvian primary teachers}

\author{
Frank Villegas \\ Pontificia Universidad Católica del Perú \\ fvillegas@pucp.edu.pe
}

Recibido: 20-03-2017

Aprobado: 01-10-2017

1. El presente artículo se basa en la tesis para optar el grado de magíster en Cognición, Aprendizaje y Desarrollo que el autor realizó con la asesoría del Dr. Santiago Cueto. 


\title{
Resumen
}

Pese a que el número de estudios sobre las creencias de los docentes se ha incrementado en los últimos años, aún es poco lo que se sabe a nivel internacional sobre aquellas vinculadas con la escritura y su enseñanza. Por un lado, para fines de esta investigación, se distinguen dos maneras sobre cómo las personas pueden entender la escritura: como proceso y como producto. De otro lado, se distinguen dos orientaciones relacionadas con la enseñanza de la escritura: enfoque comunicativo y enfoque normativo. En este contexto, este estudio tuvo como propósitos indagar qué características docentes se asocian con las creencias de los maestros sobre la enseñanza de la escritura y describir cómo se relacionan las creencias sobre la escritura de los maestros con sus creencias sobre la enseñanza de la escritura. Para el análisis, se consideró las respuestas de 3629 maestros de educación primaria de todas las regiones de Perú, quienes respondieron diversos cuestionarios en la Evaluación Muestral 2013, conducida por el Ministerio de Educación. Se encontró que las características sociodemográficas (edad, experiencia laboral, formación docente) no se relacionan o se relacionan muy poco (tal es el caso del sexo) con el enfoque de enseñanza de los maestros. Asimismo, se halló que en los maestros coexisten dos enfoques de enseñanza teóricamente incompatibles: el enfoque comunicativo y el enfoque normativo. Además, mediante ecuaciones estructurales, se encontró que las creencias sobre la escritura son buenos predictores del enfoque de enseñanza de los maestros. Estos hallazgos tienen implicancias para la formación docente.

Palabras clave: cognición docente, expresión escrita, creencias docentes

\begin{abstract}
Although the number of studies of teachers' beliefs has increased in recent years, little is still known on an international level about teachers' beliefs regarding writing and how it is taught. Two different ways in which persons can understand writing are distinguished: as a process and as a product. At the same time, two different approaches to teaching of writing are distinguished: the communicative approach and the normative approach. In this context, this study was intended to find out what teaching characteristics are associated with teachers' beliefs about teaching of writing, and to describe how teachers' beliefs about writing are related with their beliefs about the way it is taught. For the analysis, were considered responses by 3629 primary teachers from all regions of Peru, who replied to several questionnaires during the administration of the Ministry of Education's 2013 Sample-based Assessment. It was found that socio-demographic characteristics (age, work experience, teacher training) are not related or are weakly related (sex) with teachers' teaching approach. It was also found that two theoretically incompatible teaching approaches coexist among teachers: the communicative approach and the normative approach. Also, through structural equations, it was found that beliefs about writing are good predictors of the teachers' teaching approach. These findings have implications for teacher training.
\end{abstract}

Keywords: teacher cognition, writing, teachers' beliefs. 


\section{Creencias sobre la escritura y su enseñanza en maestros peruanos de educación primaria}

\section{Introducción}

La escritura es una competencia fundamental para la vida ciudadana. Por un lado, el aprendizaje de esta competencia posibilita la comunicación en situaciones que van más allá de la oralidad, sin importar el tiempo o el espacio. Así, las personas amplían sus posibilidades de comunicación. De otro lado, es una herramienta importante para el aprendizaje dentro y fuera de la escuela. Además, hace posible la expresión creativa de las personas (Graham, 2006; Rijlaarsdam et al., 2012). En este contexto, el rol de los docentes es crucial para el desarrollo de esta competencia en las escuelas, debido a que la implementación del currículo se encuentra a su cargo. No obstante, es poco lo que se sabe hasta el momento a nivel nacional e internacional sobre un elemento crucial en su toma de decisiones: sus creencias sobre la escritura y sobre su enseñanza (Borg, 2006). A continuación, se presenta un marco conceptual que permitirá, al finalizar esta sección, justificar el problema y plantear las preguntas que guiarán este estudio.

\subsection{Creencias}

El estudio de las creencias tiene más de cincuenta años y, desde entonces, ha existido una amplia variedad de aproximaciones. Aunque en los últimos veinte años el número de estudios se ha incrementado notoriamente (Ashton, 2015), aún no existe un consenso al definir el concepto de creencia. Pajares (1992) fue uno de los primeros en señalar esta falta de consenso y cómo este concepto es usado de manera intercambiable con una larga lista de términos, como por ejemplo- concepciones, disposiciones, juicios, teorías implícitas, teorías explícitas o teorías personales. De manera similar, veinte años después, Fives y Buehl (2012) plantearon que esta falta de consenso ha limitado su potencial exploratorio y predictivo.

De acuerdo con la revisión de la literatura realizada por Skott (2015), a pesar de la falta de acuerdo sobre una definición de creencia, sí existe suficiente consenso sobre cuatro aspectos centrales: (i) usualmente, es usada para describir un constructo mental de carácter individual; (ii) suele ser vinculada con aspectos cognitivos y afectivos de las personas; (iii) es considerada como estable en el tiempo; y (iv) es asumida como influyente en las prácticas docentes. Los tres primeros aspectos serán desarrollados en esta sección; y el cuarto aspecto, en la sección de esta introducción referida a las creencias docentes.

Las creencias suelen ser consideradas como juicios individuales sobre la verdad de una proposición (Pajares, 1992; Richardson, 1996; Ashton, 2015). A partir de esta definición, la relación entre las creencias y el conocimiento puede ser entendida de dos maneras. Por un lado, debido a que las creencias permiten 
comprender el mundo, algunos autores las han considerado como parte del conocimiento de una persona (Alexander, Schallert \& Hare, 1991). Incluso, en esta línea, estos términos han sido usados como sinónimos (Kagan, 1990; Fenstermacher, 1994). Desde otra perspectiva, se asume que el conocimiento se basa en el consenso social y no en el juicio personal. Por tal motivo, se considera al conocimiento y a la creencia como dos constructos diferentes (Ashton, 2015). Para fines de esta investigación, se considerará que el conocimiento y la creencia son constructos diferentes para, de esta manera, hacer explícitas sus diferencias.

A diferencia del conocimiento, las creencias se forman a partir de aspectos cognitivos y afectivos (Ashton, 2015; Pajares, 1992). Al respecto, Nespor (1987) señala que las creencias se basan principalmente en los sentimientos y en las evaluaciones subjetivas que las personas realizan de la realidad. Por otra parte, a diferencia de las emociones, las creencias son más estables en tiempo y menos intensas (McLeod, 1992). Más adelante, se brindarán mayores detalles sobre las variables que pueden influir en la estabilidad de las creencias en el tiempo.

\subsection{Creencias sobre la escritura}

A la fecha, existen pocas investigaciones respecto de las creencias de las personas sobre la escritura (Norman \& Spencer, 2005; White \& Bruning, 2005; Baaijen, Galbraith \& de Glopper, 2014; Limpo \& Alves, 2014; Sanders-Reio et al., 2014). Aunque estos estudios no se centraron únicamente en maestros en formación o en servicio, son antecedentes que permiten contextualizar la manera como se indagan este tipo de creencias en este estudio.

White y Bruning (2005) plantearon una distinción entre dos tipos de creencias sobre la escritura: «escritura como transmisión» y «escritura como transacción». La primera orientación supone que escribir bien implica transferir información de una o más fuentes autorizadas a los lectores sin que las ideas del autor del texto se reflejen en el texto escrito. De otro lado, la segunda orientación supone que escribir bien implica mostrar altos niveles de compromiso en el proceso de escritura, debido a que el propósito de la escritura es construir un texto de manera crítica y personal. Como consecuencia de este estudio, los investigadores encontraron que estas creencias predecían la calidad de los textos producidos por los participantes (estudiantes universitarios): tener una mayor puntuación en la creencia «escritura como transacción» se relacionaba con una mayor calidad en el texto producido, mientras que tener una mayor puntuación en la creencia «escritura como transmisión» se relacionaba con una menor calidad en el texto producido. Cabe señalar que, en estudios más recientes, la distinción propuesta por White y Bruning ha sido retomada por Sanders (2014), y por Baaijen, Galbraith y de Glopper (2014).

A partir del análisis de las autobiografías de 59 maestros en formación, 34 I Norman y Spencer (2005) encontraron -de manera predominante- dos tipos 
de creencia sobre la escritura: «escritura como un talento» y «escritura como una competencia maleable». La primera orientación supone que los buenos escritores lo son como consecuencia de tener un don natural que algunos poseen y otros no. De otro lado, de acuerdo con la segunda orientación, la escritura es una competencia que se puede desarrollar como consecuencia de la enseñanza y el trabajo duro. Como resultado de este estudio, los investigadores encontraron que las creencias sobre la escritura de los participantes se relacionaban con sus creencias sobre la enseñanza de la escritura.

De manera similar a Norman y Spencer, pero desde una perspectiva cuantitativa, Limpo y Alves (2014) plantearon dos tipos de orientaciones sobre la maleabilidad de la competencia de escritura al estudiar las creencias de estudiantes de quinto y sexto grado de primaria. Por un lado, la primera orientación supone que la competencia de escritura no está sujeta a mejoras. En este sentido, cualquier intento para desarrollarla sería considerado inútil. Por otro lado, la segunda orientación supone que la competencia de escritura sí se puede desarrollar. De acuerdo con los autores, si los estudiantes consideran que la escritura es una competencia que se puede desarrollar, entonces, trabajarán duro y buscarán estrategias para mejorar.

Sobre la base de la revisión de las teorías de la composición escrita (Bereiter \& Scardamalia, 1987; Graham, 2006; Kellogg, 2008; Hayes, 2012; Rijlaarsdam et al., 2012), para fines de este estudio, se distinguen dos orientaciones sobre cómo las personas pueden entender la escritura: «escritura como proceso» $\mathrm{y}$ «escritura como producto». La primera orientación supone la necesidad de dedicar tiempo y esfuerzo a la planificación y a la revisión de las ideas del texto con el fin de escribir adecuadamente. Por su parte, la segunda orientación valora principalmente el producto escrito y no el proceso de escritura. En este sentido, supone que un buen escritor debe producir un texto de calidad al primer intento y sin cometer errores. Por ello, planificar o revisar constituyen actividades innecesarias para un buen escritor. Cabe destacar que esta distinción acerca de las creencias sobre la escritura es una nueva línea de investigación propuesta por la Oficina de Medición de la Calidad de los Aprendizajes (UMC) del Ministerio de Educación de Perú (Minedu, 2016c) que puede continuar en el futuro.

\subsection{Creencias docentes}

Diversas estudios muestran cómo las creencias de los maestros se relacionan tanto con sus prácticas como con las oportunidades para aprender y los logros de sus estudiantes (Woolfolk, Davis \& Pape, 2006). Dada la importancia de las creencias en la cognición de los maestros, en las últimas décadas, muchos estudios internacionales se han concentrado en estudiar las creencias docentes sobre la naturaleza del aprendizaje y la enseñanza.

Las creencias de los docentes se forman como consecuencia de su experiencia personal, su formación profesional y su colaboración con colegas 
(Skott, 2015). Luego de que se forman, estas son consideradas estables en el tiempo y resistentes al cambio, aun así se muestren contradictorias con la razón, la escolaridad y la experiencia (Pajares, 1992; Fives \& Buehl, 2012). No obstante, diversos estudios han reportado que las creencias cambian durante la formación inicial docente, o como consecuencia de la reflexión y de la experiencia docente (Levin, 2015).

De otro lado, las creencias de los docentes se relacionan profundamente con sus prácticas y las experiencias de aprendizaje de sus estudiantes (Fives \& Gill, 2015). Cada nueva información o experiencia de los maestros es interpretada a la luz de sus creencias. Dichas creencias son usadas por los docentes para establecer cuáles son las tareas a realizar o los problemas de su experiencia cotidiana. En ese sentido, las creencias guían la práctica docente (Fives \& Buehl, 2012).

Esta investigación tiene como propósito indagar sobre las creencias de los docentes sobre la enseñanza de la escritura. En función de ello, la siguiente sección reúne los principales antecedentes en esta línea de investigación.

\subsection{Creencias docentes sobre la enseñanza de la escritura}

Existen pocas investigaciones respecto de las creencias de los docentes sobre la enseñanza de la escritura. En este contexto, los estudios de Poulson (2001), Graham (2002), y Gaitas y Alves (2014) son importantes antecedentes, que tuvieron una aproximación cuantitativa y recogieron la información sobre las creencias de los docentes a través de cuestionarios.

Sobre la base de las respuestas de 225 maestros de educación primaria en Inglaterra, el estudio de Poulson et al. (2001) distinguió 3 tipos de orientaciones vinculadas con la enseñanza de la escritura. La primera orientación priorizaba aspectos de forma (como ortografía y caligrafía); la segunda, aspectos de estructura (como la organización de las oraciones); la tercera, a diferencia de las dos primeras, priorizaba la comunicación. Este estudio reportó que el tipo de creencias de los maestros sobre la enseñanza de la escritura se relaciona con su formación profesional, el número de años de experiencia profesional y el tipo de programa de formación docente. Otro hallazgo fue que estas creencias se asociaron con sus prácticas pedagógicas. Por un lado, aquellas que conciben a la escritura como un medio para comunicarse se correlacionaron positivamente con actividades de escritura creativa. Por otro lado, aquellas creencias relacionadas con una aproximación tradicional de la enseñanza de la escritura (énfasis en aspectos como ortografía o caligrafía) correlacionaron positivamente con actividades como el copiado de frases u oraciones.

El estudio de Graham et al. (2002) se realizó sobre la base de las respuestas de 150 maestros de primer grado de educación primaria en Estados Unidos. Como consecuencia del procesamiento de los datos, el análisis factorial reveló tres factores predominantes. El primero reflejó una aproximación tradicional 36 I a la enseñanza de la escritura, con énfasis en aspectos de forma; el segundo 
priorizó la enseñanza explícita tanto del proceso de escritura como de aspectos de forma; y el tercero, la comunicación. De otro lado, este estudio también mostró que actividades como la escritura creativa, la enseñanza explícita de estrategias, la socialización de los textos y el trabajo colaborativo correlacionaron positivamente con creencias que conciben a la escritura como un medio de expresión. Asimismo, reportó que actividades como la enseñanza explícita de la ortografía y gramática correlacionaron positivamente con creencias vinculadas a una aproximación tradicional a la enseñanza de la escritura.

Por último, el estudio de Gaitas y Alves (2014) se realizó sobre la base de 225 respuestas de maestros de primaria de Portugal. El análisis factorial reveló dos factores predominantes. El primero reflejó una aproximación a la enseñanza de la escritura que enfatiza aspectos formales como la ortografía y la gramática. De otro lado, el segundo factor evidenció una aproximación que entiende la escritura como un medio de expresión. Adicionalmente, esta investigación reportó que actividades como el copiado, el dictado, el uso de fichas de ortografía y de gramática, y la escritura de temas asignados por el maestro correlacionaron positivamente con una orientación tradicional a la enseñanza de la escritura. Asimismo, mostró que actividades como la planificación y la revisión de textos, la producción de cuentos y de textos informativos se correlacionaron positivamente con una orientación que privilegia la comunicación al momento de enseñar a escribir.

Como consecuencia de la investigación, se pueden distinguir dos marcadas orientaciones en relación con la enseñanza de la escritura. La primera prioriza la comunicación durante la enseñanza, mientras que la segunda enfatiza la enseñanza de aspectos de forma y, de esta manera, refleja una aproximación tradicional a la enseñanza de la escritura. Para fines de este estudio, se considerará esta distinción y se denominará a la primera orientación «enfoque comunicativo» y a la segunda, «enfoque normativo» o tradicional.

\subsection{El enfoque comunicativo en el currículo peruano}

En los últimos años, un importante número de países latinoamericanos ha implementado cambios curriculares que privilegian la enseñanza y la evaluación por competencias. En este marco, el área de Comunicación de estos países privilegia, a nivel de currículo intencional, el desarrollo de la competencia comunicativa de sus estudiantes (Laboratorio Latinoamericano de la Calidad de la Educación, 2013). En el Perú, la política curricular para la enseñanza de la lengua en la educación básica se basa en el enfoque comunicativo (Minedu, 2003, $2009,2016 c$ ). Este enfoque tiene como propósito que los estudiantes logren un manejo eficiente de la lengua al expresar sus ideas de manera oral, al leer y al producir textos. A diferencia de una aproximación tradicional de la enseñanza de la lengua, el centro de los aprendizajes no es la ortografía y la gramática.

Aunque desde hace varios años los documentos curriculares peruanos plantean el enfoque comunicativo, estas propuestas han sido plasmadas 
de distintas maneras. El Diseño Curricular Nacional (DCN) 2003, junto con su actualización del año 2009, representó un cambio significativo en el enfoque pedagógico del currículo escolar; sin embargo, tuvo problemas de construcción interna (excesiva densidad, falta de coherencia y ambigüedad) y no logró convertirse en un documento de apoyo para los docentes (Minedu, 2014). Frente a esta situación, el Ministerio de Educación inició el proceso de desarrollo de un nuevo sistema curricular, el cual estaba basado en un Marco Curricular Nacional, Mapas de Progreso y Rutas de Aprendizaje. No obstante, este proceso de cambio se detuvo, a pesar de los avances que ya se habían logrado. En su lugar, se plantearon modificaciones al DCN 2009 a través de la Resolución Ministerial 199-2015 (Minedu, 2015), que estuvo vigente durante 2015 y 2016. Para el año 2017, el Ministerio de Educación ha oficializado el Currículo Nacional, el cual se propone recoger las experiencias y los avances realizados desde 2010 (Minedu, 2016c).

Con respecto a la competencia vinculada a la escritura, el DCN, en sus dos versiones, planteó la importancia de que esta, dentro de la escuela, responda a un propósito comunicativo, $y$, de esta manera, se propicie el aprendizaje del proceso de escritura y sus distintas etapas (Minedu, 2005, 2009). En términos generales, los últimos documentos curriculares (Minedu, 2015b, 2016c) coinciden en que, al finalizar su escolaridad, los estudiantes estén en la posibilidad de escribir distintos tipos texto, con coherencia, con cohesión y un uso apropiado de los aspectos formales del lenguaje.

Por lo antes mencionado, según los lineamientos planteados por la política curricular peruana, se esperaría que en los docentes responsables del área de Comunicación predominen (i) creencias sobre la escritura como proceso y (ii) creencias vinculadas con el enfoque comunicativo.

\subsection{Planteamiento del problema y preguntas de investigación}

A pesar de la importancia del aprendizaje de la escritura para la vida social, los resultados de las evaluaciones de logro de aprendizaje implementadas por la UMC muestran que la mayoría de los estudiantes peruanos no logra los aprendizajes esperados para el grado en la competencia de escritura. Por un lado, la Evaluación Muestral 2013 revela que solo el 13,5 \% de los estudiantes de sexto grado de primaria produce cuentos según las características esperadas para el grado (Minedu, 2016a). De otro lado, en segundo de secundaria, la prueba de escritura aplicada en el marco de la Evaluación Censal de Estudiantes 2015 da cuenta de que solo el 12,6\% de los estudiantes logra los aprendizajes esperados (Minedu, 2016b). Estos resultados ponen en evidencia las dificultades del sistema educativo peruano para implementar su propuesta curricular.

En la misma línea, diversas investigaciones muestran la poca coherencia entre la política curricular y las prácticas docentes relacionadas con la escritura. Por ejemplo, el estudio de Cueto et al. (2006) encontró que la mayoría (casi

el $80 \%$ ) de las tareas propuestas por los maestros del área de Comunicación 
en 20 escuelas de Lima y de Ayacucho requerían únicamente memorización y trabajo mecánico para ser resueltas. De otro lado, otros estudios (Zavala, 2002; Ames, 2006; González, Eguren, de Belaunde, 2017) señalan que la escritura no es promovida en las escuelas como un medio de expresión. Por el contrario, las actividades de escritura que realizan los estudiantes se limitan al copiado de lo que el profesor dicta o escribe en la pizarra. Asimismo, indican que, cuando los estudiantes escriben, los docentes enfatizan aspectos formales como la gramática y la ortografía. Dichos estudios sugieren que predomina un enfoque tradicional de la enseñanza de la escritura en los maestros peruanos, en lugar del enfoque comunicativo.

En este contexto, el estudio de las creencias docentes sobre la enseñanza de la escritura es relevante, en la medida que, sobre la base de estas creencias, los maestros interpretan la política curricular e implementan el currículo. Asimismo, conocer qué variables se asocian a estas creencias brinda evidencia para la toma de decisiones sobre la formación inicial o en servicio de los docentes peruanos del área de Comunicación. Sobre esta base, se determinaron dos preguntas que guían este estudio: ¿Qué características docentes se asocian con las creencias sobre la enseñanza de la escritura en los maestros peruanos de educación primaria? y ¿cómo se relacionan las creencias sobre la escritura y las creencias sobre la enseñanza de la escritura en los maestros peruanos de educación primaria?

\section{Método}

\subsection{Base de datos}

Los datos que se utilizan fueron producidos por la Evaluación Muestral (EM) 2013. Esta evaluación de logros de aprendizaje fue implementada por la Oficina de Medición de la Calidad de los Aprendizajes (UMC) del Ministerio de Educación de Perú. La UMC seleccionó de manera aleatoria una muestra de estudiantes de sexto grado de educación primaria, pertenecientes a 3120 escuelas de todas las regiones del Perú. Esta muestra siguió un diseño probabilístico por conglomerados bietápico y estratificado. Como parte de la aplicación de la EM, también se invitó a participar a los docentes del área de Comunicación de los estudiantes evaluados y se les pidió responder un cuestionario. Así, se configuró quiénes serían los participantes de este estudio.

Los participantes fueron 3629 docentes de sexto grado de educación primaria de distintas regiones del Perú, con edades que van desde los 22 hasta los 70 años (media $=44,7$ y desviación estándar $[\mathrm{DE}]=9,53$ ), y una experiencia docente entre 0 y 45 años (media $=18,10$ y $\mathrm{DE}=9,5$ ). En relación con el tipo de gestión de las escuelas en las que laboraban, 3074 docentes trabajaban en escuelas estatales ( $84,7 \%)$; y 555, en escuelas no estatales (15,3\%). Sobre el área, 2451 docentes trabajaban en escuelas urbanas $(67,7 \%)$, mientras que 1178 , 
en escuelas rurales (32,5\%). En cuanto a género, del total de los participantes, 1541 son varones $(42,5 \%)$; y 2063, mujeres (56,8\%).

A partir de lo antes mencionado, es necesario tomar en consideración que los resultados de este estudio no tienen representatividad estadística de la población de docentes de sexto grado de educación primaria del área de Comunicación, dado que el diseño muestral tuvo como propósito representar a los estudiantes y no a los docentes. Pese a ello, este estudio ofrece información sobre docentes del área de Comunicación de escuelas con características diversas de todas las regiones del Perú.

\subsection{Medición}

\section{Ficha de datos sociodemográficos}

Para describir a cada participante, se tomaron en consideración variables vinculadas con información sociodemográfica. La siguiente tabla presenta cada una de estas variables con una breve descripción.

Tabla 1.Variables sociodemográficas

\begin{tabular}{|c|c|}
\hline Participante & Descripción \\
\hline Sexo & $\begin{array}{l}1=\text { Mujer } \\
0=\text { Hombre }\end{array}$ \\
\hline Edad & Número en años \\
\hline Experiencia docente & Número en años \\
\hline Formación inicial $^{2}$ & $\begin{array}{l}\text { 1= Estudió en un instituto superior pedagógico (ISP) } \\
2=\text { Estudió pregrado en una universidad } \\
3=\text { Estudió en un programa de profesionalización docente } \\
4=\text { Estudió otra carrera }\end{array}$ \\
\hline
\end{tabular}

Fuente: Elaboración propia

\section{Enfoque de enseñanza}

Se usó una versión adaptada de la escala Writing Orientation de Graham (2002). Esta versión ${ }^{3}$ fue elaborada por especialistas del equipo de Comunicación y de Factores Asociados de la UMC y consta de doce ítems. Para medir

2. Para este análisis, se crearon tres variables ficticias. Asimismo, se consideró como grupo de referencia al grupo de maestros que estudió una carrea distinta a Educación.

40 I. Este cuestionario se puede encontrar en $<$ http://umc.minedu.gob.pe/evaluacionmuestral-2013/>. 
este constructo, se pidió a los maestros marcar en una escala Likert según cuán identificados se sentían respecto de afirmaciones sobre la enseñanza de la escritura. Esta escala consta de cinco opciones que van desde el «Totalmente en desacuerdo» hasta el «Totalmente de acuerdo».

Sobre la base de la evidencia proporcionada por los análisis factoriales exploratorios y la revisión de literatura, se partió del supuesto de la existencia de dos factores latentes: (i) enfoque comunicativo (e.g. "Los estudiantes aprenderán las reglas ortográficas poco a poco, a medida que practican su escritura y escriben mensajes a los demás») y (ii) enfoque normativo (e.g. «Antes de que los estudiantes comiencen a escribir un texto, es muy importante recordarles que cuiden su ortografía»). El análisis factorial confirmatorio ${ }^{4}$ (AFC) corroboró la existencia de estos dos factores con índices adecuados de bondad de ajuste $(\mathrm{CFI}=0,972, \mathrm{TLI}=0,950, \mathrm{RMSEA}=0,066: \mathrm{IC} \text { al 90\% }=[0,058-0,073])^{5}$. De otro lado, las cargas factoriales fueron significativas $(\mathrm{p}<0,001)$ y estuvieron por encima de 0,30, un valor adecuado según Kline (2016). En este sentido, las cargas de los ítems del primer factor fluctuaron entre 0,556 y 0,636 , mientras que las cargas del segundo factor fluctuaron entre 0,468 y 0,883 . Sobre la confiabilidad, el índice de fiabilidad compuesta del enfoque comunicativo es 0,736 ; y el de enfoque normativo, 0,708 .

Tal como se realizó en estudios previos (Poulson et al., 2001; Graham et al., 2002; Gaitas \& Alves, 2014), se corroboró que el constructo «enfoque de enseñanza» es multidimensional. Por un lado, el primer factor -enfoque comunicativo- refiere a la orientación de los maestros que prioriza la comunicación durante la enseñanza de la escritura y, al mismo tiempo, considera la ortografía como un contenido que progresivamente se aprende conforme los estudiantes usan la escritura como un medio de expresión. Por otro lado, el segundo enfoque -enfoque normativo- refiere a la orientación de los maestros que prioriza la enseñanza de aspectos de forma (como la ortografía, la gramática y la caligrafía) durante la enseñanza de la escritura.

\section{Creencias sobre la escritura}

Se usó un cuestionario elaborado por la UMC, el cual consta de diez ítems. Para medir este constructo, se pidió a los maestros marcar en la escala Likert según cuán identificados se sentían respecto de ciertas afirmaciones sobre la escritura. En este caso, 1 equivalía a «definitivamente, no»; y 8, a «definitivamente, sí».

Sobre la base de la evidencia proporcionada por los análisis factoriales exploratorios y la revisión de literatura, se partió del supuesto de la existencia de

4. En el caso del cuestionario sobre enfoque de enseñanza y creencias sobre la escritura, en esta sección, se presentan los resultados de los análisis realizados por el autor del artículo en relación con las evidencias de validez y de confiabilidad.

5. RMSEA = raíz cuadrada media del error de aproximación; CFI = índice de ajuste comparativo; TLI = índice de Tucker-Lewis; IC = intervalo de confianza. 
dos factores latentes: (i) creencias de la escritura como proceso (e.g. «Siempre reviso lo que acabo de escribir, pues podría haber hecho algo mal») y (ii) creencias de la escritura como producto (e.g. "Una persona que no escribe bien un texto al primer intento no es un buen escritor»). El AFC corroboró la existencia de estos factores con índices aceptables de bondad de ajuste (CFI $=0,939$; TLI $=0,905 ;$ SRMR $=0,047$; RMSEA $=0,051$ : IC al 90\% $=[0,045-0,056])$. Asimismo, las cargas factoriales fueron significativas y estuvieron por encima de 0,30 en casi todos los casos. En este sentido, las cargas correspondientes al primer factor fluctuaron entre 0,256 y 0,720, mientras que las cargas del segundo factor oscilaron entre 0,438 y 0,688 . Cabe mencionar que se mantuvo un ítem con baja carga factorial, debido a que su eliminación no implicaba una ganancia respecto de la bondad de ajuste. Sobre la confiabilidad de las escalas, el índice de fiabilidad compuesta de «escritura como proceso» es 0,723 y el de «escritura como producto» es 0,652 .

El AFC permitió corroborar el supuesto de que el constructo «creencias sobre la escritura» es multidimensional y que reúne dos factores. Ambos factores refieren a dos tipos de orientación de las personas respecto de su propia comprensión de la escritura. El primer factor - «escritura como proceso»- refiere a la orientación que considera a los procesos de planificación y de revisión como etapas necesarias para producir un buen texto. De otro lado, el segundo factor - «escritura como producto»- alude a la orientación en la que se asume que un buen escritor produce un buen texto al primer intento, sin cometer errores, y sin la necesidad de planificar o revisar lo escrito. A diferencia del constructo mencionado en la sección anterior, no existen estudios previos que hayan indagado de manera similar a las creencias sobre la escritura. En este sentido, indagar las creencias sobre la escritura de esta manera es una nueva línea de investigación, propuesta por la UMC (Minedu, 2016c), que puede continuar en el futuro.

\section{Procedimiento}

Especialistas de los equipos de Evaluación y Factores Asociados de la UMC elaboraron el cuestionario a partir de una revisión bibliográfica de las teorías sobre el proceso de escritura y de la didáctica de la escritura. En este sentido, la revisión de la literatura presentada en la introducción de este artículo incluye los referentes tomados en cuenta por el equipo de la UMC. Luego, la UMC condujo una aplicación piloto de los instrumentos de la EM 2013. Al finalizar esta fase, los especialistas de la UMC realizaron diversos ajustes a los instrumentos con miras a la aplicación definitiva.

Para la aplicación definitiva de la EM, una vez identificadas las escuelas y las secciones que participarían según el diseño muestral, la UMC contrató aplicadores, quienes fueron capacitados con el fin de estandarizar los procesos. El primer día de aplicación, cada aplicador se puso en contacto con el direc42 I tor de la escuela a la que fue asignado y anunció en qué consistiría su trabajo 
durante los siguientes tres días. El cuestionario dirigido a los docentes de Comunicación fue entregado al inicio del primer día y fue recogido al finalizar el mismo día. Al culminar esta fase, se consolidó una base de datos que integra la información proveniente del cuestionario dirigido a los docentes del área de Comunicación. Finalmente, esta base fue solicitada por el responsable de este estudio y, luego de su recepción, se firmó un acuerdo de confidencialidad. En la siguiente sección, se describe en detalle el proceso de análisis de datos realizado por el autor del artículo con el propósito de enriquecer el trabajo realizado por la UMC.

\section{Análisis de los datos}

Primero, se realizó el análisis descriptivo de cada uno de los ítems del cuestionario mediante el uso del paquete estadístico SPSS, versión 22. Asimismo, se realizaron pruebas de normalidad multivariada sobre los datos del cuestionario de creencias sobre la escritura ${ }^{6}$. En este caso, se llevaron a cabo las pruebas de Mardia (1970), y de Henze y Zirkler (1990) mediante el paquete MNV de la versión 3.2.5 del software estadístico $R$.

Para evaluar la validez de las escalas, se realizaron análisis factoriales confirmatorios con el fin de corroborar su estructura factorial. Para ello, en el caso del cuestionario relacionado con el enfoque de enseñanza de los maestros, se usó el método de estimación de mínimos cuadrados ponderados robustos (WLSMV), porque es el más apropiado cuando los ítems tienen cinco o menos categorías (Kline, 2016). En el caso del cuestionario sobre las creencias sobre la escritura, el cual tiene ítems de ocho categorías, se usó el método de estimación de máxima verosimilitud con la corrección de Satorra y Benther (MLM, 2001), debido a que los datos no cumplen con el supuesto de normalidad multivariada. Estos análisis se realizaron mediante el paquete lavaan del software R.

Los análisis factoriales confirmatorios fueron evaluados sobre la base de la combinación de diversos índices de ajuste de los modelos de ecuaciones estructurales (SEM). En primer lugar, se utilizó la raíz cuadrada media del error de aproximación (RMSEA). Para el RMSEA, un valor cercano a 0,06 (Hu \& Bentler, 1999) o un límite superior de 0,07 (Steiger, 2007) son considerados buenos ajustes. Asimismo, se tomaron en consideración índices de ajustes relativos: el índice de ajuste comparativo (CFI) y el índice de Tucker-Lewis (TLI). En cuanto a estos índices, valores superiores a 0,90 indican un ajuste aceptable (Brown, 2015), mientras que valores mayores a 0,95 representan un buen ajuste (Hu \& Bentler, 1999).

6. Es necesario tomar en cuenta que, cuando los indicadores tienen cinco o más categorías, pueden considerarse como variables continuas (Bovaird \& Koziol, 2012; Raykov, 2012; Li, 2014). 
De otro lado, para analizar la confiabilidad de las escalas, se calculó el índice de fiabilidad compuesta propuesto por Raykov (1997). Este índice muestra en qué medida un conjunto de indicadores son consistentes al medir un constructo latente (Raykov, 2012). En comparación con el a de Crombach, el CR es considerado una mejor alternativa (Raykov, 2012; Brown, 2015; Kline, 2016). Dado que los constructos indagados se encuentran en una fase de exploración, se consideraron como aceptables valores de CR por encima de 0,60 (Hair et al., 2014)

Sobre la base de los AFC, se le asignó a cada docente cuatro puntajes factoriales por cada uno de los factores indagados. En este contexto, un mayor puntaje factorial revela que el docente posee más de determinado rasgo latente. El cálculo de los puntajes factoriales se realizó mediante el paquete lavaan del software estadístico R. Los datos proporcionados por el archivo de salida de $\mathrm{R}$ fueron ordenados por medio del lenguaje de programación python, versión 2.9.1. Luego, estos datos fueron llevados a una base de datos en el programa SPSS. Finalmente, por medio del SPSS, se transformaron los puntajes factoriales en puntajes $Z$.

Como el propósito de esta investigación es establecer el grado de relación entre variables, se realizaron diversos análisis. Primero, se hicieron análisis de correlación de Pearson entre las variables indagadas. Luego, se realizaron dos regresiones lineales múltiples. En el primer modelo de regresión, se consideró como variable dependiente a "enfoque comunicativo" y, en el segundo modelo, a la variable «enfoque normativo». Al respecto, es necesario mencionar que las variables contextuales (área y gestión) no fueron incluidas en los modelos de regresión múltiple, debido a que su inclusión transgrede el supuesto de independencia requerido para análisis de un solo nivel como la regresión de mínimos cuadrados ordinarios (Andréu, 2011; Heck \& Thomas, 2015).

Finalmente, se tomó en consideración a las variables de interés para plantear un modelo de ecuaciones estructurales con el propósito de conocer cómo se relacionan estas variables en simultáneo (Nachtigall, Kroehne, Funke, \& Steyer, 2003). Este último análisis se realizó con el software R. Asimismo, los resultados fueron evaluados con los índices de ajuste mencionados previamente.

\section{Resultados}

\subsection{Descriptivos}

Como parte de los análisis preliminares, se calculó los promedios al interior de cada uno de los subgrupos según las variables sociodemográficas. Esta información se presenta a continuación en la tabla 2. 
Tabla 2. Puntajes promedio según las variables sociodemográficas

\begin{tabular}{lccccccccc}
\hline \multirow{2}{*}{$\begin{array}{c}\text { Variables } \\
\text { sociodemográficas }\end{array}$} & \multicolumn{2}{c}{$\begin{array}{c}\text { Escritura } \\
\text { como proceso }\end{array}$} & \multicolumn{2}{c}{$\begin{array}{c}\text { Escritura como } \\
\text { producto }\end{array}$} & \multicolumn{2}{c}{$\begin{array}{c}\text { Enfoque } \\
\text { comunicativo }\end{array}$} & \multicolumn{2}{c}{$\begin{array}{c}\text { Enfoque } \\
\text { normativo }\end{array}$} \\
\cline { 2 - 10 } & Media & DE & Media & DE & Media & DE & Media & DE \\
\hline Sexo & & & & & & & & & \\
Hombre & $-0,09$ & 1,07 & 0,11 & 1,01 & $-0,07$ & 0,98 & $-0,02$ & 0,98 \\
Mujer & 0,07 & 0,94 & $-0,08$ & 0,98 & 0,05 & 1,01 & 0,02 & 1,02 \\
\hline Formación inicial & & & & & & & & \\
\hline Estudió en una ISP & 0,03 & 0,97 & $-0,07$ & 1,01 & $-0,01$ & 1,00 & $-0,05$ & 1,02 \\
Estudión un pregrado & 0,04 & 0,94 & $-0,12$ & 0,93 & 0,05 & 1,02 & $-0,01$ & 1,04 \\
$\begin{array}{l}\text { Profesionalización } \\
\text { docente }\end{array}$ & $-0,08$ & 1,05 & 0,23 & 0,99 & $-0,02$ & 0,98 & 0,06 & 0,95 \\
Estudió otra carrera & $-0,21$ & 1,36 & 0,26 & 1,04 & 0,26 & 1,03 & 0,38 & 0,89 \\
\hline
\end{tabular}

Nota: Los puntajes factoriales fueron transformados a puntajes Z. Esto significa que para cada variable el promedio es 0 ; y la desviación estándar, 1 . Sobre la base de esta transformación, se elaboró esta tabla. Para estimar la significancia de las diferencias entre los grupos según sexo y formación inicial respecto de enfoque comunicativo y enfoque normativo, se introdujeron estas variables en una regresión lineal múltiple. Esta información puede encontrarse en la sección de análisis principales de «Resultados».

Fuente: Elaboración propia

\subsection{Relación entre las variables}

Para conocer las relaciones existentes entre las variables indagadas, se realizaron análisis de correlación de Pearson, debido a que estas siguen una distribución normal univariada. Como indica la tabla 3 , las variables indagadas se relacionan de manera significativa. 
Tabla 3.Correlaciones entre las variables indagadas

\begin{tabular}{ccccc}
\hline \multicolumn{1}{c}{ Escala } & 1 & 2 & 3 \\
& & EC & EN & PROC \\
\hline Enfoque de enseñanza & & & & \\
\hline Enfoque comunicativo & $(1)$ & & & \\
Enfoque normativo & $(2)$ & $0,715^{\star * *}$ & & \\
\hline Creencias sobre la escritura & & & & \\
\hline Escritura como proceso & $(3)$ & $0,410^{* * *}$ & $0,231^{\star * *}$ & \\
Escritura como producto & $(4)$ & $0,053^{* *}$ & $0,375^{\star * *}$ & $0,050^{\star *}$ \\
\hline
\end{tabular}

${ }^{* * *} \mathrm{p}<0,001 ;{ }^{* *} \mathrm{p}<0,01 ;{ }^{*} \mathrm{p}<0,05$

Nota: Enfoque comunicativo $=$ EC, Enfoque normativo $=$ EN, Escritura como producto $=$ PROC

Fuente: Elaboración propia

Con respecto a las relaciones intra-escalas, «enfoque comunicativo» correlaciona de manera positiva, alta y significativa con «enfoque normativo» $(\mathrm{r}=0,715 ; \mathrm{p}<0,001)$. Por otro lado, «escritura como proceso» presenta una correlación muy baja y negativa con «escritura como producto».

En relación con los análisis entre escalas, «enfoque comunicativo» presenta una correlación moderada, positiva y significativa con «escritura como proceso» $(\mathrm{r}=0,410 ; \mathrm{p}<0,001)$ y una relación muy baja con "escritura como producto» $(\mathrm{r}=0,053 ; \mathrm{p}<0,01)$. Además, «enfoque normativo» se relaciona de manera moderada, positiva y significativa con «escritura como producto» $(\mathrm{r}=0,375$; $\mathrm{p}<0,001)$; $\mathrm{y}$, de forma baja, positiva y significativa con la «escritura como producto» $(\mathrm{r}=0,231 ; \mathrm{p}<0,001)$.

\subsection{Análisis de regresión múltiple}

Con el propósito de determinar qué variables docentes permiten explicar las creencias sobre la enseñanza de la escritura, se llevaron a cabo dos análisis de regresión múltiple con el método de pasos sucesivos. El primer análisis consideró a "enfoque comunicativo» como variable dependiente, y a las variables sociodemográficas (sexo, formación inicial, edad, experiencia laboral) y a las creencias sobre la escritura («escritura como proceso» y «escritura como producto») como variables independientes. De otro lado, el segundo análisis de regresión consideró a las mismas variables independientes y a «enfoque normativo» como variable dependiente. 
Tabla 4. Análisis de regresión múltiple que predice el enfoque comunicativo

\begin{tabular}{|c|c|c|c|c|c|}
\hline & $\mathrm{R} 2$ & $\begin{array}{c}\mathrm{R} 2 \\
\text { ajustada }\end{array}$ & $\Delta \mathrm{R}^{2}$ & $\beta$ & $\mathrm{T}$ \\
\hline Paso 1 & 0,13 & 0,13 & 0,13 & & \\
\hline Escritura como proceso & & & & $0,36^{* * *}$ & 21,11 \\
\hline Paso 2 & 0,13 & 0,13 & 0,001 & & \\
\hline Escritura como proceso & & & & $0,38^{\star * *}$ & 20,56 \\
\hline Escritura como producto & & & & $0,04^{*}$ & 1,98 \\
\hline Paso 3 & 0,13 & 0,13 & 0,001 & & \\
\hline Escritura como proceso & & & & $0,37^{* * *}$ & 20,48 \\
\hline Escritura como producto & & & & $0,04^{*}$ & 2,12 \\
\hline Sexo & & & & $0,04^{*}$ & 2,10 \\
\hline
\end{tabular}

Nota: ${ }^{* *} \mathrm{p}<0,001 ;{ }^{* *} \mathrm{p}<0,01 ;{ }^{*} \mathrm{p}<0,05$

Fuente: Elaboración propia

Como se puede apreciar en la tabla 4, en el primer paso del primer análisis de regresión, «escritura como proceso» explica el 13\% de la proporción de la variabilidad de «enfoque comunicativo». Cuando la variable «escritura como producto» es introducida en el modelo, ocurre un cambio muy pequeño en el $\mathrm{R}^{2}(0,001)$. Esto mismo ocurre en el tercer y último paso, cuando la variable sexo es introducida. Finalmente, el modelo de regresión múltiple está conformado por tres variables: «escritura como proceso», «escritura como producto» $\mathrm{y}$ «sexo» (a favor de las mujeres). Estas variables explican el 13\% de la variabilidad. Cabe señalar que las variables «edad», «experiencia laboral» y aquellas vinculadas con la formación inicial no mostraron una relación significativa con la variable dependiente, y, por ello, no se incluyen los coeficientes.

De otro lado, en relación con el segundo análisis, «escritura como producto» explica el 5\% de la variabilidad de "enfoque normativo» en el primer paso. En el segundo paso, cuando la variable «escritura como producto» es introducida, ambas variables explican el $12 \%$ de la variabilidad del modelo (tabla 5). Cabe mencionar que en este modelo las variables «sexo», «edad», «experiencia laboral» y aquellas vinculadas con la formación inicial de los maestros no mostraron una relación significativa con la variable dependiente. 
Tabla 5. Análisis de regresión múltiple que predice el enfoque normativo

\begin{tabular}{cccccc}
\cline { 2 - 6 } & $\mathrm{R} 2$ & $\begin{array}{c}\mathrm{R} 2 \\
\text { ajustada }\end{array}$ & $\Delta \mathrm{R}^{2}$ & $\beta$ & $\mathrm{T}$ \\
\hline $\begin{array}{l}\text { Paso 1 } \\
\text { Escritura como producto }\end{array}$ & 0,05 & 0,05 & 0,05 & & \\
\hline Paso 2 & 0,12 & 0,12 & 0,08 & & \\
Escritura como producto & & & & $0,30^{\star * *}$ & 20,56 \\
Escritura como proceso & & & & $0,28^{\star * *}$ & 1,98 \\
\hline
\end{tabular}

Nota: ${ }^{* *} \mathrm{p}<0,001 ;{ }^{* *} \mathrm{p}<0,01 ;{ }^{*} \mathrm{p}<0,05$

Fuente: Elaboración propia

\subsection{Modelo de ecuaciones estructurales}

Como consecuencia de la revisión de la literatura, se planteó un modelo hipotético (figura 1) sobre la relación entre las variables indagadas. Dado que las variables sociodemográficas no aportan o aportan muy poco para explicar la variabilidad del enfoque comunicativo y el enfoque normativo, estas no fueron incluidas en el modelo hipotético.

Figura 1. Modelo hipotético

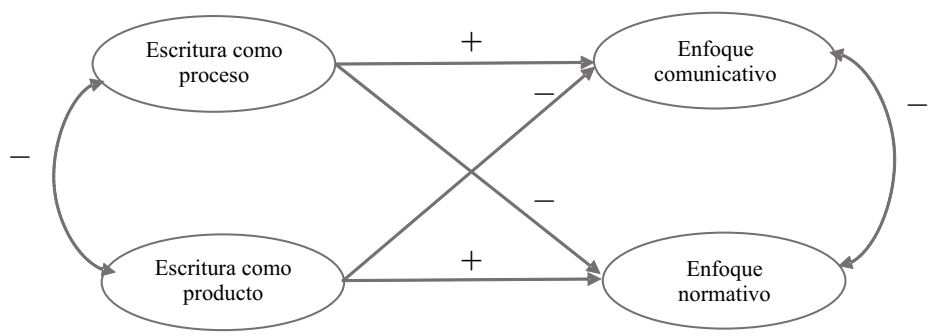

Fuente: Elaboración propia

El modelo hipotetizado tuvo un ajuste aceptable a los datos (RMSEA = 0,038: IC al 90\% = [0,035 - 0,040]; CFI = 0,924; TLI =0,905; SRMR =0,042). Aunque el CFI y el TLI muestran valores aceptables, los valores del RMSEA y el SRMR son excelentes. Adicionalmente, la mayoría de los pasajes resultaron significativos.

Como muestra la figura 2, solo la variable «escritura como proceso» predice «enfoque comunicativo» $(\beta=0,35 ; \mathrm{p}<0,001)$, dado que el pasaje de «escritura como producto» no es significativo $(\beta=0,02$; n.s.). Asimismo, «escri48 I tura como producto» es un mejor predictor $(\beta=0,32$; $p<0,001)$ de «enfoque 
normativo» en comparación con «escritura como proceso» $(\beta=0,19 ; \mathrm{p}<0,001)$. En cuanto a la relación entre las variables «escritura como proceso» $\mathrm{y}$ "escritura como producto», esta es muy baja y no significativa $(-0,04 ; p=0,161)$. De otro lado, la relación entre «enfoque comunicativo» $\mathrm{y}$ «enfoque normativo» es $0,65(\mathrm{p}<0,001)$.

Figura 2. Modelo de ecuaciones estructurales

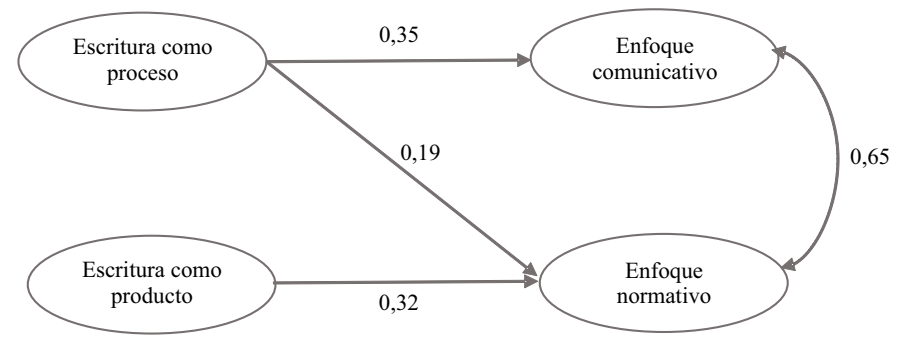

RMSEA=0,038; CFI $=0,924 ;$ TLI $=0,905 ;$ SRMR $=0,042$.

Nota: La figura muestra solo aquellos pasajes que resultaron significativos.

Fuente: Elaboración propia

\section{Discusión}

Esta investigación tuvo un doble propósito. Por un lado, se buscó determinar qué características docentes se asocian con las creencias docentes sobre la enseñanza de la escritura en los maestros peruanos de educación primaria. Por otro lado, se apuntó a describir cómo se relacionan sus creencias sobre la escritura con sus creencias sobre la enseñanza de la escritura. En este marco, se discutirán los resultados obtenidos en tres secciones: (i) sobre los resultados obtenidos, (ii) sobre el significado práctico de los resultados, y (iii) sobre las fortalezas y las limitaciones del estudio.

\subsection{Sobre los resultados obtenidos}

En relación con el primer objetivo de este estudio, los resultados obtenidos muestran que las características sociodemográficas no se relacionan (edad, experiencia laboral y formación inicial) o se relacionan muy poco (sexo) con el enfoque de enseñanza de los maestros peruanos. Sobre estos primeros resultados, por un lado, llama mucho la atención que el número de años de experiencia docente no contribuya a explicar la variabilidad del enfoque comunicativo. Debido a que este enfoque es parte de la política curricular peruana (Minedu, 2003, 2009, 2016d), se esperaría que una mayor experiencia docente represente también un mayor alineamiento con el enfoque planteado por el Ministerio de 
Educación. No obstante, a diferencia de otros estudios (Young \& Cook, 2004; Alger, 2009), no se aprecia esta tendencia.

Alger (2009) les pidió a los participantes de su estudio elegir una metáfora que se aproxime lo más posible a sus creencias sobre la enseñanza en tres situaciones: al inicio de su carrera, en el presente y en términos ideales. Un hallazgo de este estudio fue que el $63 \%$ de los maestros cambió su orientación de enseñanza. En el caso de los maestros con mayor experiencia laboral, de acuerdo con lo que reportaron, iniciaron su carrera docente con una orientación de enseñanza centrada en el maestro y, luego, cambiaron esta por una orientación centrada en el estudiante. Por su parte, Young y Cook (2004), luego de analizar los textos escritos por dieciocho estudiantes de educación, encontraron que para los maestros en formación la experiencia de interactuar con estudiantes es una fuente de motivación para cambiar su sistema de creencias con el propósito de atender de una mejor manera a las demandas de sus estudiantes.

A diferencia de lo que ocurre en otros contextos, los resultados obtenidos en este estudio sugieren que la formación en servicio de los maestros peruanos no contribuye a un progresivo alineamiento de sus creencias con la política curricular. Esto podría interpretarse de tres maneras distintas, las cuales no son excluyentes entre sí. En primera instancia, este hallazgo podría indicar, tal como señala Cuenca (2008), que no se está garantizando una formación en servicio que responda de manera adecuada a las necesidades de los maestros peruanos. En segunda instancia, podría indicar que los temas vinculados a didáctica de la escritura no han recibido la atención necesaria de parte de los programas de formación docente en servicio. Finalmente, se podría argumentar que los continuos cambios en los documentos curriculares, como se comentó en la introducción, no han contribuido a que las creencias de los maestros peruanos se alineen con el enfoque propuesto por el Ministerio de Educación. En todo caso, mayores estudios son necesarios para corroborar estas hipótesis o plantear nuevas.

De otro lado, llama la atención, dado que es un resultado inesperado, que participar en un programa de profesionalización docente o estudiar pedagogía en una ISP o en una universidad, en comparación con estudiar una carrera distinta a Educación, no represente un valor agregado al explicar el enfoque de enseñanza de los maestros. En esta situación, se esperaría lo contrario: que estudiar pedagogía posibilite una mayor comprensión y alineamiento con la política curricular, tal como la investigación ha puesto en evidencia en otros contextos distintos al peruano (Polikoff, 2013). En este sentido, lo anterior podría considerarse como una manifestación de la baja calidad de la formación inicial docente (en ISP o en universidad) y de los programas de profesionalización docente (Cuenca \& Stojnic, 2008).

Esta investigación tuvo como segundo propósito indagar cómo se relacionan las creencias sobre la escritura y las creencias sobre la enseñanza de la escritura en los maestros peruanos. Al respecto, uno de los hallazgos es que se encontró una alta relación $(0,65)$ entre el puntaje obtenido por los maestros en 
la subescala enfoque comunicativo y la subescala enfoque normativo. Esto sugiere que ambos enfoques coexisten en el sistema de creencias de los maestros peruanos, a pesar de que son incompatibles desde un punto de vista teórico. Este hallazgo es consistente con la evidencia proporcionada por otras investigaciones relacionadas con la enseñanza de la escritura en educación primaria (Pressley, Rankin, \& Yokoi, 1996; Graham et al., 2002; Gaitas \& Alves, 2014). Adicionalmente, es consistente con los hallazgos de otros estudios que muestran que en los maestros pueden coexistir enfoques pedagógicos teóricamente incompatibles (Verjovsky \& Waldegg, 2005; Snider \& Roehl, 2007; Cheng et al., 2009).

La organización del sistema de creencias es psicológica y no necesariamente lógica (Pajares, 1992; Fives \& Buehl, 2012). Por tal motivo, es concebible que en un mismo docente coexistan dos orientaciones sobre la enseñanza inconsistentes desde un punto de vista lógico. En el contexto peruano, Moreano et al. (2008) encontraron que en los participantes de su estudio también coexistían dos enfoques incompatibles: el enfoque para la enseñanza de las matemáticas planteado por el Ministerio de Educación y un enfoque tradicional. Esta coexistencia se reflejó en la discrepancia entre el discurso de los docentes y sus prácticas: el discurso mostraba adhesión al nuevo enfoque y las prácticas reflejaban el enfoque tradicional.

Sobre la base de lo antes mencionado, futuros estudios podrían explorar en los maestros peruanos la relación de coexistencia de los dos enfoques de enseñanza planteados en este estudio. Asimismo, podrían indagar sobre cómo esta relación se refleja sus prácticas pedagógicas. De esta manera, como hizo Moreano et al. (2008), se podría establecer qué creencias prevalecen finalmente en el aula de clases.

Otro hallazgo relacionado con el segundo propósito de este estudio es que se encontró que las creencias sobre la escritura son buenos predictores del enfoque de enseñanza de los maestros. Por un lado, se encontró que escritura como proceso predice el enfoque comunicativo $(\beta=0,35)$. Por otro lado, se halló que escritura como producto es un mejor predictor del enfoque normativo $(\beta=0,32)$ en comparación con escritura como proceso $(\beta=, 19)$. Como indica Borg (2006), son pocos los estudios que han explorado la cognición de los maestros sobre la enseñanza de la escritura. En este contexto, son aún menos los estudios (Norman \& Spencer, 2005) que han indagado sobre la relación entre las creencias sobre la escritura y las creencias sobre la enseñanza de la escritura. Por tal motivo, el hallazgo mencionado constituye un aporte teórico al estudio de las creencias docentes.

Existen creencias que constituyen un obstáculo para nuevos enfoques de enseñanza, así también existen creencias que pueden facilitar el desarrollo profesional (Skott, 2015). En este sentido, de acuerdo con la evidencia, el que los maestros consideren necesarias las etapas de planificación y de revisión en su propio proceso de escritura facilita el que ellos puedan apropiarse del enfoque comunicativo para la enseñanza de la escritura. De otro lado, el que 
los maestros asuman que un buen escritor produce un buen texto al primer intento, sin cometer errores y sin la necesidad de planificar o revisar es un obstáculo para su desarrollo profesional, dado que esta creencia facilita en mayor medida el enfoque normativo para la enseñanza de la escritura, el cual es un enfoque no deseado según la política curricular peruana.

Desde una aproximación cualitativa, Norman y Spencer (2005) obtuvieron un hallazgo similar al estudiar 59 autobiografías de maestros en formación. Estos investigadores encontraron que los maestros en formación que consideraban a la escritura como un talento que solo algunos poseen no encontraban en la enseñanza una influencia positiva para el desarrollo de la producción escrita. Por el contrario, los maestros en formación que consideraban que la escritura es una habilidad "que se puede desarrollar» veían que una buena enseñanza y el trabajo «duro» podían influir positivamente en el desarrollo de la competencia escrita.

Tanto este estudio como el de Norman y Spencer (2005) sugieren que las creencias sobre la enseñanza de la escritura no deben estudiarse de manera aislada, sino más bien en relación con otras creencias. En la misma línea que plantean Fives, Lacatena y Gerard (2015), es necesario reconocer que los maestros manejan diferentes creencias de manera simultánea. Por ello, futuros estudios podrían indagar qué otras creencias permiten explicar las creencias de los maestros sobre la enseñanza de la escritura.

\subsection{Sobre el significado práctico de los resultados}

Diversos autores señalan la necesidad y la efectividad de que en las escuelas los maestros brinden oportunidades a sus estudiantes para atravesar las distintas etapas del proceso de escritura para producir textos que respondan a sus necesidades y a sus intereses (Graves, 1989; Bomer, 1995; Atwell, 1998; Calkins, 2003; Lattimer, 2003; Rijlaarsdam et al., 2005; Graham \& Perin, 2007; Graham \& Sandmel, 2011). Para ello, es necesario que los maestros desarrollen creencias sobre la escritura como proceso.

Los resultados de esta investigación constituyen un insumo importante para la formación docente. En primer lugar, la formación inicial o en servicio de los maestros, además de considerar los aspectos propios de la didáctica de la escritura, debería ocuparse también de las creencias de los docentes sobre la escritura. En este sentido, es necesario que los maestros o futuros maestros participen durante su formación de experiencias significativas y sostenidas en el tiempo que les permitan reflexionar y, de ser el caso, cuestionar sus creencias sobre la escritura. Naturalmente, para que esto ocurra, los centros de formación, en lugar de asumir que los estudiantes ya saben escribir (Carlino, 2005), deberían tener como meta el desarrollo de la competencia escrita de los maestros o futuros maestros.

En segundo lugar, la formación de los maestros debería aspirar a que 52 I ellos conceptualicen sus propios procesos de cognición. Como se mencionó 
previamente, en los maestros peruanos, coexisten dos enfoques de enseñanza teóricamente incompatibles. En este contexto, una formación que brinde a los maestros las herramientas necesarias para reflexionar sobre sus propias creencias podría permitir que estas cambien $y$, a su vez, influyan positivamente en sus prácticas docentes.

En tercer lugar, dado que ha iniciado la implementación del Currículo Nacional, el cual estará vigente desde 2017, el sistema educativo peruano cuenta con la oportunidad de realizar los esfuerzos necesarios para promover el alineamiento de las creencias y las prácticas de los docentes con la política curricular.

\subsection{Sobre las fortalezas y las limitaciones del estudio}

En las secciones previas, se han discutido los principales hallazgos de esta investigación. Por su parte, esta sección tiene como propósito comentar estos hallazgos a partir de sus fortalezas y sus limitaciones metodológicas.

En la EM 2013, la UMC recogió información sobre las creencias docentes por medio de cuestionarios, lo cual permitió tener una aproximación al sistema de creencias de 3629 maestros de distintas regiones del Perú. Aunque el uso de cuestionarios es la estrategia más usada en los estudios sobre creencias docentes, lo más recomendable es usar diversas estrategias de recojo de información (Schraw \& Olafson, 2015). Entre otras estrategias, se pueden realizar entrevistas, observaciones de clase, portafolios o análisis de metáforas. En este sentido, los resultados de este estudio deben ser considerados como una aproximación a las creencias de los docentes peruanos, la cual necesariamente debe ser complementada y profundizada con fututos estudios cuyo diseño sea de tipo cualitativo o mixto.

Otro aspecto a comentar es el relacionado con los instrumentos. Estos instrumentos tuvieron como propósito medir constructos que se encuentran en fase de exploración. Esto se evidencia en la cantidad de ítems de las escalas. En este sentido, aunque se contó con una cantidad suficiente de ítems para realizar los AFC, una mayor cantidad de los mismos permitiría representar de una mejor manera los constructos indagados y, al mismo tiempo, mejorar los índices de ajuste de las escalas. Por tal motivo, futuros estudios podrían enriquecer las escalas elaboradas por la UMC y, de esta manera, continuar con una línea de investigación sobre la que se ha trabajado muy poco a nivel nacional e internacional.

Por último, es necesario mencionar que una futura investigación, con los mismos datos analizados en este artículo, debería considerar las variables contextuales de área y de gestión en los análisis. Para dicho propósito, un modelo de ecuaciones estructurales multinivel permitiría tener una mejor aproximación a la relación que existe entre las variables (Heck \& Thomas, 2015). 


\section{Nota biográfica}

Frank Villegas

Es licenciado en Educación Primaria de la Pontificia Universidad Católica del Perú (PUCP) y magíster en Cognición, Aprendizaje y Desarrollo por la misma universidad. Actualmente, es docente en la Facultad de Educación y la Escuela de Posgrado de PUCP. Sus principales áreas de interés y especialización son didáctica de la lectura y la escritura, educación matemática, evaluación educativa, cognición docente y desarrollo cognitivo. 


\section{Referencias}

Atwel, N. (1998). In the middle. New York: Heinemann.

Alexander, P. A., Schallert, D. L. \& Hare, V. C. (1991). Coming to terms: How Researchers in learning and literacy talk about knowledge. American Educational Research Association, 3, 315-343.

Alger, C. L. (2009). Secondary teachers' conceptual metaphors of teaching and learning: Changes over the career span. Teaching and Teacher Education, 25(5), 743-751.

Ames, P. (2006). Las brechas invisibles: Desafíos para una equidad de género en la educación. Lima: Instituto de Estudios Peruanos.

Andréu, J. (2011). El análisis multinivel: Una revisión actualizada en el ámbito sociológico. Metodología de Encuestas, 13, 161-176.

Ashton, P. (2015). Historical overview and theoretical perspectives of research on teachers' beliefs. In H. Fives \& M. G. Gill (Eds.), International handbook of research on teachers' beliefs (pp. 31-47). New York: Routledge.

Baaijen, V. , Galbraith, D. \& de Glopper, K. (2014). Effects of writing beliefs and planning on writing performance. Learning and Instruction, 33(2014), 81-91.

Behizadeh, N. (2014). Mitigating the dangers of a single story: Creating largescale writing assessments aligned with sociocultural theory. Educational Researcher, 43(3), 125-136.

Bereiter, C., \& Scardamalia, M. (1987). The psychology of written composition. Hillsdale, NJ: Erlbaum.

Bomer, R. (1995). Time for meaning. New York: Heinemann.

Borg, S. (2006). Teacher cognition and language education. London: Continuum.

Bovaird, J., \& Koziol, N. (2012). Measurement models for ordered-categorical indicators. En R. Hoyle (Ed.), Handbook of structural equation model (pp. 475-509). The Guilford Press.

Brown, T. (2015). Confirmatory factor analysis for applied research. New York: The Guilford Press.

Brown, T., \& Moore, M. (2012). Confirmatory factory analysis. In Handbook of structural equation modeling (pp. 361-379). New York: The Guilford Press.

Calkins, L. (1986). Didáctica de la escritura. Buenos Aires: Aique.

(2003). The nuts and bolts of teaching writing. New York: Heinneman.

Carlino, P. (2005). Escribir, leer y aprender en la universidad. Buenos Aires: Fondo de Cultura Económica.

Cheng, M., Chang, K., Tang, S. \& Cheng, A. (2009). Pre-service teacher education students' epistemological beliefs and their conceptions of teaching. Teaching and Teacher Education, 25, 319-327. 
Cohen, J. (1988). Statistical power analysis for the behavioral sciences. Statistical power analysis for the behavioral sciences ( $2^{\mathrm{a}}$ edición). New York: Routledge.

Cuenca, R., \& Stojnic, L. (2008). La cuestión docente en el Perú. Buenos Aires: FLAPE.

Cueto, S., Ramírez, C., León, J. \& Azañedo, S. (2006). Oportunidades de aprendizaje y rendimiento en comunicación integral de estudiantes en tercer y cuarto grado de primaria en Lima y Ayacucho. Los desafíos de la escolaridad en el Perú. Estudios sobre los procesos pedagógicos, los saberes previos y el rol de las familias (pp. 13-78). Lima: Grade.

Fenstermacher, G. D. (1994). Chapter 1: The knower and the known: The nature of knowledge in research on teaching. Review of Research in Education, 20(1), 3-56.

Fives, H., \& Buehl, M. (2012). Spring cleaning for the «messy» construct of teachers' beliefs: What are they? Which have been examined? What can they tell us? APA Educational Psychology Handbook. Volumen 2: Individual Differences and Cultural and Contextual Factors (pp. 471-499). Washington: American Psychological Association.

Fives, H., \& Gill, G. (Eds.) (2015). International handbook of research in teachers' beliefs. New York: Routledge.

Fives, H., Lacatena, N. \& Gerard, L. (2015). Teachers' beliefs about teaching (and learning). In International handbook of research on teachers' beliefs (pp. 249-265). New York: Routledge.

Gaitas, S. \& Alves, M. (2014). Relationships between primary teachers' beliefs and their practices in relation to writing instruction. Research papers in education (mayo 2015), 1-14.

Graham, S. (2006). Writing. In P. A. Alexander (Ed.), Handbook of educational psychology (pp. 457-478). New Jersey: Lawrence Erlbaum Associates.

González, N, Eguren, M, de Belaunde, C. (2017). Desde el aula: Una aproximación a las prácticas pedagógicas del maestro peruano. Lima: Instituto de Estudios Peruanos.

Graham, S., Harris, K. R., MacArthur, C. \& Fink, B. (2002). Primary grade teachers' theoretical orientations concerning writing instruction: Construct validation and a nationwide survey. Contemporary educational psychology, 27(2), 147-166.

Graham, S. \& Perin, D. (2007). A meta-analysis of writing instruction for adolescent students. Journal of Educational Psychology, 99(3), 445-476. http://doi.org/10.1037/0022-0663.99.3.445

Graham, S. \& Sandmel, K. (2011). The process writing approach: A metaanalysis. The Journal of Educational Research, 104(6), 396-407.

56 I Graves, D. (1989). Experiment with fiction. Toronto: Heinemann. 
Hair, J. F., Sarstedt, M., Hopkins, L., G. Kuppelwieser, V. \& Kuppelwieser, V. G. (2014). Partial Least Squares Structural Equation Modeling (PLS-SEM): An emerging tool in business research. European Business Review, 26(2), 106-121.

Hamp-Lyons, L. (2011). Writing assessment: Shifting issues, new tools, enduring questions. Assessing Writing, 16(1), 3-5.

Hayes, J. (1996). A New Framework for Understanding Cognition And Affect in Writing. In S. Levy \& C. Ransdell (Eds.), The science of writing: Theories, methods, individual differences and applications (pp. 1-27). New Jersey: Erlbaum.

(2011). Kinds of knowledge-telling: Modeling early writing development. Journal of Writing Research, 3(2), 73-92.

(2012). Modeling and Remodeling Writing. Written Communication, 29(3), 369-388.

Heck, R. \& Thomas, S. (2015). An introduction to multilevel modeling techniques: MLM and SEM approaches using Mplus. New York: Routledge.

Henze, N. \& Zirkler, B. (1990). A class of invariant consistent tests for multivariate normality. Communications in Statistics - Theory and Methods, 19(10), 3595-3617.

Hernández, R., Fernández, C. \& Baptista, P. (2014). Metodología de la investigación. México D.F.: Mc Graw Hill Education.

Hu, L. \& Bentler, P. M. (1999). Cut off criteria for fit indexes in covariance structure analysis: Conventional criteria versus new alternatives. Structural Equation Modeling: A Multidisciplinary Journal, 6(1), 1-55.

Huot, B. \& O’Neill, P. (2009). Assesing writing. New York: National Council of Teachers of English.

Kagan, D. (1990). Ways of evaluating teacher cognition: Inferences concerning the goldilocks principle. Review of Educational Research, 60, 419-469. (1992). Implications of research on teachers' beliefs. Educational Psychologist, 27, 65-90.

Kellogg, R. (2008). Training writing skills: A cognitive developmental perspective. Journal of Writing Research, 1(1), 1-26.

Kline, R. (2016). Principles and practice of structural equation modeling. New York: The Guilford Press.

Korkmaz, S., Goksuluk, D. \& Zararsiz, G. (2014). MVN: An R package for assessing multivariate normality. The R Journal, 6(2013), 151-162.

Laboratorio Latinoamericano de la Calidad de la Educación. (2013). Análisis curricular. Santiago de Chile: Oficina Regional de Educación para América Latina y el Caribe.

Lattimer, H. (2003). Thinking trough genre. Portland: Stenhouse Publishers. 
Levin, B. (2015). The development of teachers' beliefs. In H. Fives \& G. Gill (Eds.), International handbook of research on teachers' beliefs (pp. 48-65). New York: Routledge.

Li, C.-H. (2014). The performance of MLR, USLMV, and WLSMV estimation in structural regression models with ordinal variables. Ann Arbor: Michigan State University.

Limpo, T., \& Alves, R. (2014). Implicit theories of writing and their impact on students' response to a SRSD intervention. British Journal of Educational Psychology, 84(4), 571-590.

Mardia, K. (1970). Measures of multivariate skewness and kurtosis with applications. Biometrika, 57(3), 519-530. http://doi.org/10.1093/ biomet/57.3.519

McLeod, D. (1992). Research on affect in mathematics education: A reconceptualization. In Handbook of research on mathematics teaching and learning (pp. 575-596). New York: Macmillan.

Ministerio de Educación. (2005). Diseño curricular nacional. Lima: Ministerio de Educación.

de Educación.

(2009). Diseño curricular nacional. Lima: Ministerio (2014). Marco curricular nacional, segunda versión. Lima: Ministerio de Educación.

(2015). Resolución Ministerial 199-2015 (2015).

Lima.

(2016a). ¿Qué logran nuestros estudiantes al finalizar la primaria? Lima: Ministerio de Educación.

(2016b). ¿Qué logran nuestros estudiantes en Escritura? Lima: Ministerio de Educación.

(2016c). ¿Qué logros de aprendizaje en Escritura muestran los estudiantes al finalizar la primaria? Informe de evaluación de Escritura en sexto grado - 2013. Lima: Ministerio de Educación.

Educación.

Moreano, G., Asmad, U., Cruz, G. \& Cuglieva, G. (2008). Concepciones sobre la enseñanza de matemática en docentes de primaria de escuelas estatales. Revista de Psicología PUCP, 2, 299-336.

Nachtigall, C., Kroehne, U., Funke, F. \& Steyer, R. (2003). (Why) should we use SEM? Pros and cons of structural equation modeling. Methods of Psychological Research, 8(2), 1-22.

Nespor, J. (1987). The role of beliefs in the practice of teaching. Jounal of Curriculum Studies, 19(mayo 2015), 317-328. 
Newell, A. \& Simon, H. (1972). Human problem solving. NJ: Prentice-H.

Norman, K. A. \& Spencer, B. H. (2005). Our lives as writers: Examining preservice teachers' experiences and beliefs about the nature of writing and writing instruction. Teacher Education Quarterly, 32(1), 25-40.

Pajares, F. (1992). Teachers' beliefs and educational research: Cleaning up a messy construct. Review of Educational Research, 62(3), 307-332. http:// doi.org/10.3102/00346543062003307

Confidence and competence in writing: The role of self-efficacy, outcome expectancy, and apprehension. Atlanta: American Educational Research Association.

Pajares, F., \& Valiante, G. (1999). Grade level and gender differences in the writing self-beliefs of middle school students. Contemporary Educational Psychology, 24(4), 390-405. http://doi.org/10.1006/ceps.1998.0995

Polikoff, M. S. (2013). Teacher education, experience, and the practice of aligned instruction. Journal of Teacher Education, 64(3), 212-225.

Poulson, L., Avramidis, E., Fox, R., Medwell, J. \& Wray, D. (2001). The theoretical beliefs of effective teachers of literacy in primary schools: An exploratory study of orientations to reading and writing. Research Papers in Education, 16(3), 271-292.

Pressley, M., Rankin, J. \& Yokoi, L. (1996). A survey of instructional practices of primary teachers nominated as effective in promoting literacy. The Elementary School Journal, 96(4), 363-384.

Raykov, T. (1997). Estimation of composite reliability for congeneric measures. Applied Psychological Measurement, 21(2), 173-184. http://doi. org/0803973233

(2012). Scale construction and development using structural equation modeling. In R. Hoyle (Ed.), Handbook of structural equation model (pp. 472-494). New York: The Guilford Press.

Richardson, V. (1996). The role of attitudes and beliefs in learning to teach. In J. Sikula (Ed.), Handbook of research on teacher education (pp. 102-109). New York: Macmillan.

Rijlaarsdam, G., Van den Bergh, H., Couzijn, M., Janssen, T., Braaksma, M., Tillema, M., Raedts, M. (2012). Writing. APA Educational Psychology Handbook, Vol. 3: Application to Learning and Teaching, 3, 189-227. http:// doi.org/10.1037/13275-009

Sanders-Reio, J., Alexander, P., Reio, T. \& Newman, I. (2014). Do students' beliefs about writing relate to their writing self-efficacy, apprehension, and performance? Learning and Instruction, 33, 1-11.

Satorra, A. \& Bentler, P. (2001). A scaled difference chi-square test statistic for moment structure analysis. Psychometrika, 66(4), 507-514. 
Schraw, G. \& Olafson, L. (2015). Assessing teachers' beliefs. In International handbook of research on teachers' beliefs (pp. 87-105). New York: Routledge.

Skott, J. (2015). The promises, problems, and prospects of research on teachers' beliefs. In H. Fives \& M. M. Buehl (Eds.), International handbook of research on teachers' beliefs (pp. 13-30). New York: Routledge.

Snider, V. \& Roehl, R. (2007). Teachers' beliefs about pedagogy and related issues. Psychology in the Schools, 48, 873-886.

Steiger, J. H. (2007). Understanding the limitations of global fit assessment in structural equation modeling. Personality and Individual Differences, 42(5), 893-898.

Thompson, A. G. (1992). Teachers' beliefs and conceptions: A synthesis of the research. Handbook of research on mathematics teaching and learning, 127146. Recuerado de http://psycnet.apa.org/psycinfo/1992-97586-007

Verjovsky, J. \& Waldegg, G. (2005). Analyzing beliefs and practices of a mexican high school biology teacher. Journal of Reseach in Science Teaching, 42, 465-491.

White, M. J. \& Bruning, R. (2005). Implicit writing beliefs and their relation to writing quality. Contemporary Educational Psychology, 30(2), 166-189.

Woolfolk, A., Davis, H., \& Pape, S. (2006). Teachers' knowledge, beliefs, and thinking. In P. A. Alexander \& P. H. Winne (Eds.), Handbook of educational psychology (pp. 715-737). New Jersey: Lawrence Erlbaum Associates.

Young, J. R. \& Cook, P. F. (2004). Face-to-face with children. Journal of Curriculum Studies, 36, 341-360.

Zavala, V. (2002). Las prácticas letradas escolares en castellano y en quechua: El uso de adivinanzas y narraciones en el aula. Desencuentros con la escritura. Escuela y comunidad en los Andes peruanos (pp. 1-37). Lima: Red para el Desarrollo de las Ciencias Sociales en el Perú. 